\title{
Peripheral Inflammation Increases the Damage in Animal Models of Nigrostriatal Dopaminergic Neurodegeneration: Possible Implication in Parkinson's Disease Incidence
}

\author{
A. Machado, ${ }^{1,2}$ A. J. Herrera, ${ }^{1,2}$ J. L. Venero, ${ }^{1,2}$ M. Santiago,,${ }^{1,2}$ R. M. De Pablos, ${ }^{1,2}$ \\ R. F. Villarán, ${ }^{1,2}$ A. M. Espinosa-Oliva, ${ }^{1,2}$ S. Argüelles, ${ }^{1,2}$ M. Sarmiento, ${ }^{1,2}$ \\ M. J. Delgado-Cortés, ${ }^{1,2}$ R. Mauriño, ${ }^{1,2}$ and J. Cano ${ }^{1,2}$ \\ ${ }^{1}$ Departamento de Bioquímica y Biología Molecular, Facultad de Farmacia, Universidad de Sevilla, 41012 Sevilla, Spain \\ ${ }^{2}$ Instituto de Biomedicina de Sevilla (IBiS), Hospital Universitario Virgen del Rocío/CSIC/Universidad de Sevilla, \\ 41013 Sevilla, Spain
}

Correspondence should be addressed to A. Machado, machado@us.es

Received 12 November 2010; Accepted 21 February 2011

Academic Editor: Gilles J. Guillemin

Copyright () 2011 A. Machado et al. This is an open access article distributed under the Creative Commons Attribution License, which permits unrestricted use, distribution, and reproduction in any medium, provided the original work is properly cited.

Inflammatory processes described in Parkinson's disease (PD) and its animal models appear to be important in the progression of the pathogenesis, or even a triggering factor. Here we review that peripheral inflammation enhances the degeneration of the nigrostriatal dopaminergic system induced by different insults; different peripheral inflammations have been used, such as IL- $1 \beta$ and the ulcerative colitis model, as well as insults to the dopaminergic system such as 6-hydroxydopamine or lipopolysaccharide. In all cases, an increased loss of dopaminergic neurons was described; inflammation in the substantia nigra increased, displaying a great activation of microglia along with an increase in the production of cytokines such as IL- $1 \beta$ and TNF- $\alpha$. Increased permeability or disruption of the BBB, with overexpression of the ICAM-1 adhesion molecule and infiltration of circulating monocytes into the substantia nigra, is also involved, since the depletion of circulating monocytes prevents the effects of peripheral inflammation. Data are reviewed in relation to epidemiological studies of PD.

\section{Introduction}

Parkinson's disease (PD) is the second most common agingrelated neurodegenerative disease after Alzheimer's disease (AD). The main symptom of PD is a movement disorder called Parkinsonism (muscle rigidity, akinesia, and resting tremor) caused by dopamine (DA) deficiency in the striatum due to DA neuron degeneration in the substantia nigra (SN). Although a small percentage of PD is familial (fPD), most is sporadic (sPD), associated with aging and with no hereditary history. Aetiology of PD probably involves both environmental agents and genetic risk factors [1-3]. The implication of inflammatory process in PD is accepted, since many inflammatory marks have been described in PD and its animal models (for a review of neuroinflammation in PD, see [4]). Consequently, neuroinflammation is now thought to be fundamental for, or even a triggering factor of, the progression of PD pathogenesis.
Activated microglia and reactive human leukocyte antigen-DR (HLA-DR)-positive microglia were found in the SN pars compacta (SNpc) $[5,6]$ in the postmortem analysis of PD patients. Immunohistochemical studies have shown many activated microglial cells in neurotoxin-treated SNpc in various animal PD models [7], suggesting the presence of inflammatory processes [8-12]. Moreover, levels of proinflammatory substances such as cyclooxygenase 2 $(\mathrm{COX} 2)$ and cytokines including interleukine-1 beta (IL$1 \beta$ ), interferon-gamma (IFN- $\gamma$ ), and tumour necrosis factoralpha $(\mathrm{TNF}-\alpha)$, are found to be high in postmortem PD brains [13-17]. Similarly, 6-hydroxydopamine (6-OHDA) and 1-methyl-4-phenyl-1,2,3,6-tetrahydropyridine (MPTP) animal PD models showed increased levels of these inflammatory mediators $[10,18-21]$, which are secreted from microglia, neurons, and astrocytes [22-25]. Therefore, these molecules may be actively involved in disease progression. 
Several studies have tried to correlate polymorphisms in the promoters of several cytokine genes to the risk of developing PD [26-31]. Pattarini et al. [32] have shown protection against MPTP toxicity-measured as attenuation of dopamine depletion in the striatum-after genetic ablation of either TNF- $\alpha$ or its receptors Tnfrsf1 and Tnfrsf2 [18, 21, 33, 34 , although neither genetic ablation nor pharmacological manipulation of TNF- $\alpha$ prevents neuronal loss in the SNpc $[18,35,36]$. On the contrary, Il-6 knockout mice are more sensitive to MPTP toxicity [37], which could be in agreement with the neuroprotective effect described for IL6 [38]. Clinical studies on chronic users of nonsteroidal anti-inflammatory drugs (NSAIDs) suggest that some of these agents could lower the incidence of PD [39-42]. However, no such association was found in other studies $[43,44]$, although this preventive effect has been described in experiments with animals [45-47]. In the MPTP model of PD [48], the inactivation of microglia also showed to be neuroprotective.

Animal models of degeneration of the nigrostriatal dopaminergic system have been developed by intranigral injection of proinflammogens [11, 49-60]. Furthermore, other features support the implication of inflammation in the development or progression of PD. Chronic traumatic brain injury associated with boxing has been etiologically linked to PD with the well-known "punch-drunk syndrome" or "dementia pugilistica" that sometimes develops in boxers as a result of long-term subclinical concussions [61-66]. This is in agreement with the fact that inflammation through microglial activation accompanies the CNS tissue's response to injury (for review see Loane and Byrnes [67]).

The brain is considered an immunologically privileged organ, free from immune reactions, since it is protected by the blood-brain barrier (BBB). However, accumulating findings have revealed that immune responses can occur in the brain, especially because of microglial activation. These cells are known to produce proinflammatory cytokines and a relationship with the peripheral system has been suggested. Moreover, it is known that neurovascular functions are altered in aging and neurodegenerative diseases, leading to abnormal states such as increased BBB permeability, decreased nutrient supply, faulty clearance of toxic molecules, and failure of enzymatic function [68]. Moreover, several studies on PD patients and animal models suggest a pathogenic link between $\mathrm{BBB}$ disruption and DA neuronal death. Positron emission tomography (PET) and histological studies on PD patients revealed dysfunction of the BBB transport system [69] as well as alteration of blood vessels in the midbrain of PD patients [70]. In addition, levels of vascular endothelial growth factor (VEGF) and pigment epithelium-derived factor (PEDF) that induce structural changes in blood vessels were higher in PD patients and the MPTP model [71]. Interestingly, injecting VEGF in the $\mathrm{SN}$ disrupted $\mathrm{BBB}$ and induced DA neuronal death in the ventral mesencephalon [72]. In addition, increased BBB permeability was observed in the MPTP [73] and lipopolysaccharide (LPS) models of PD [74]. These results indicate that $\mathrm{BBB}$ disruption is somehow related to neuronal cell death and neuroinflammation in PD. Moreover, the presence of $\mathrm{T}$ lymphocytes in the midbrain of $\mathrm{PD}$ patients suggests that the potential role of infiltrated peripheral cells is related to the pathogenesis of PD [75]. In the model of LPS-induced inflammation, neutrophils and monocytes infiltrate into the $\mathrm{SN}$ region, where they play an important role in neuroinflammation [76]. Brochard et al. [77] reported that many CD4- and CD8-positive cells were detected postmortem in $\mathrm{PD}$ patients. In particular, the cytotoxic effects of $\mathrm{T}$ cells showed that $\mathrm{CD} 4$-deficient mice were resistant to MPTP neurotoxicity in the SN. In addition, the presence of Iba-1 positive cells in disrupted blood vessels indicates that neuroinflammation might contribute to the infiltration of peripheral immune cells and leakage of the $\mathrm{BBB}$ in the SN. Taken together, these results suggest that penetration of immune cells plays an important role in the degeneration of DA neurons in PD.

Peripheral inflammation could also have consequences on the degenerative process of DA neurons. There are many pathological circumstances in which peripheral inflammation is a common symptom. Epidemiological studies have shown that incidence of idiopathic PD is about 50\% lower in chronic users of NSAIDs or COX inhibitors than in age-matched nonusers $[39,40,78]$. This could be related to inflammation inhibition in the CNS, but also to the inhibition of peripheral inflammation. Moreover, the role of peripheral inflammation in different neurodegenerative diseases has become evident in recent years. The probability of suffering $\mathrm{AD}$ doubles in elderly individuals exposed to systemic inflammation [79]. Furthermore, induction of a systemic inflammatory response led to reactivation in animal models of multiple sclerosis [80]. Strang [81] described the increased prevalence of peptic ulcer prodromal to idiopathic Parkinsonism. This was independently produced by Helicobacter or not [82]. Systemic inflammation sensitized microglia to switch to an overactivated proinflammatory state in a model of prion disease [83]. Here, we review the possibility that peripheral inflammation could enhance the degeneration of the nigrostriatal dopaminergic system and thus it would be involved in the incidence of PD.

\section{Inflammation Increases the Degeneration of the Dopaminergic System Induced by an Insult}

First, we must note that inflammation is capable of increasing the degeneration of the dopaminergic system induced by different insults.

2.1. In Vitro Studies. Gao et al. [84, 85] have shown that low doses of neurotoxin (rotenone or MPTP) and inflammogen LPS synergistically induced a progressive and selective degeneration of dopaminergic neurons in mesencephalic neuronglia cultures. They showed that glia is required for this effect; in addition, they also showed that the effect was produced by a synergistic increase in $\mathrm{NO}$ and the superoxide free radical produced by NADPH oxidase. Similar results were described by Long-Smith et al. [86] using the cytokinerich conditioned medium (CM) from LPS-treated rat 
glial-enriched cortical cultures and 6-OHDA. They also showed that IL- $1 \beta$ in the CM mediated the increase in neuronal death since IL-1 receptor (IL-1R1) was located in dopaminergic neurons and its blockade prevented this effect. However, the authors also pointed out that many other cytotoxic factors such as TNF- $\alpha$ - and indeed some cytoprotective ones, may also be in the medium. Zhang et al. [87] also showed that combinations of $\mathrm{MnCl}_{2}$ and LPS, at minimally effective concentrations when used alone, induced synergistic and preferential damage to DA neurons in rat primary neuron-glia cultures. These authors also showed that this effect could be produced by a significant increase in TNF- $\alpha$ and IL- $1 \beta$ release along with the increased production of reactive oxygen species (ROS) and NO. These effects were attenuated by pretreatment with anti-inflammatory agents, such as minocycline and naloxone.

2.2. In Vivo Studies.. Koprich et al. [88] induced a nontoxic inflammation in the SN, injecting a non-DA-toxic dose of LPS within the SN followed by injecting a low dose of 6OHDA in the striatum, demonstrating that dopaminergic cell loss increased significantly. The authors identified IL$1 \beta$ as a potential mediator of the effect, and were able to overcome it by the administing an IL-1R1 antagonist. Similar results have been also described by Godoy et al. [19] who used a similar method, also showing that inflammation induced by a low dose of LPS in SN produced a significant increase in the degeneration of DA neurons in $\mathrm{SN}$ induced by 6-OHDA compared with 6-OHDA alone. Moreover, this effect was overcome by dexamethasone (DEX), a well-known anti-inflammatory steroid. These results show that inflammation is able to enhance the degeneration of dopaminergic neurons induced by several insults.

\section{Peripheral Inflammation Enhances the Degeneration of the Nigrostriatal Dopaminergic System}

It is important to know whether peripheral inflammation, a very common health problem, could affect the degeneration of nigrostriatal dopaminergic neurons. Godoy et al. [19] induced a peripheral-like inflammatory state by injecting an adenoviral vector expressing IL- $\beta$ (or $\beta$-galactosidase as control) in the tail vein. This model was used to study the effect of peripheral inflammation on the nigrostriatal dopaminergic neurodegeneration induced by injecting 6OHDA in the striatum. The authors found a statistically significant decrease in the number of TH-positive cells in the SN of the animals treated with 6-OHDA/Ad IL1biv in comparison with the other groups. These data showed that systemic IL-1 expression exacerbates, but does not directly elicit, neurodegeneration in the SN. This effect is produced by an increase in inflammation in $\mathrm{SN}$ as has been pointed out by the great reactivation of microglia (stage 4 morphology) found along with other parameter studies. This is the first description of the influence of peripheral inflammation on the degeneration of nigrostriatal dopaminergic system induced by an insult (6-OHDA). Similarly, Mangano and
Hayley [89], injecting low amounts of LPS in the SN and the administration of the pesticide paraquat (which has been reported to provoke DA loss) showed a greater loss of THpositive neurons in $\mathrm{SN}$ after two days.

Villarán et al. [90] studied the effect of peripheral inflammation on the degeneration of the nigrostriatal dopaminergic system induced by injecting LPS within the SN. The Ulcerative Colitis model (UC, one of the two major forms of gastrointestinal dysfunction) induced by dextran sodium sulphate (DSS) [91] was used as peripheral inflammation model; dextran sulphate provides an easy and well-characterized model that shares most features of human UC at structural, ultrastructural and clinical levels [92], including peripheral inflammation. The authors found a decrease in the number of TH-positive neurons in the animals with LPS-treated UC, doubling that found in animals treated with LPS. These results are in agreement with those described by Pott Godoy et al. [93], who studied the effect of peripheral inflammation (intravenous injection of adenovirus expressing IL- $1 \beta$ or $\beta$-galactosidase) on central inflammation (injecting adenovirus expressing IL- $1 \beta$ into the striatum). They found that chronic, systemic IL- $1 \beta$ expression exacerbated the neurodegeneration induced by IL- $1 \beta$ expression in the $\mathrm{SN}$.

\section{Mechanisms by Which Peripheral Inflammation Could Enhance the Degeneration of the Nigrostriatal Dopaminergic System Induced by Insults}

As has been described by Gao et al. $[84,85]$ in studies using primary mesencephalic neuron-glia cultures as in vitro model of $\mathrm{PD}$, participation of microglia is required for the induction of the synergistic neurotoxicity induced by inflammation (LPS) on the toxic effect of MPTP or rotenone. This suggests that inflammation (as the reactivation of microglia and secretion of many proinflammatory compounds) could be the cause of the synergistic process. In this context, they described that the release of superoxide free radical and the production of intracellular ROS was synergistic. Since this effect does not occur when NADPH oxidase-deficient (gp91phox-/-) mice were used, they also showed that it was catalyzed by NADPH oxidase, an enzyme that seems to be a major source of extracellular superoxide production in microglia. This proposal is in agreement with Godoy et al. [19] who described that stage 4 microglia and MHC II expression were associated with the exacerbation of neurodegeneration and motor symptoms. Similarly to Koprich et al. [88], these authors proposed that microglial activation towards a proinflammatory phenotype that increases IL- $1 \beta$ secretion is responsible for the synergistic effect. They pointed out that IL- $1 \beta$ is the cause of this effect, since glucocorticoid treatment and specific IL-1 inhibition overcome these effects. This proposal is also supported by an in vitro study in which IL-1 directly exacerbated 6-OHDA-triggered dopaminergic toxicity. This is also supported by Koprich et al. [88], who showed that the systemic administration of IL-1ra was able to reverse 
the vulnerability produced by LPS and therefore eliminate the contribution of 6-OHDA to DA cell death. This is in agreement with Godoy et al. [19], who also proposed that IL- $1 \beta$ is responsible for the synergistic effect seen in the animal model of chronic systemic inflammation produced by injecting an adenoviral vector expressing IL1 $\beta$ (or $\beta$ galactosidase as control) in the tail vein. They concluded that IL- $1 \beta$ could be acting directly on neurons, and also indirectly through NO. Furthermore, this is also in agreement with Ferrari et al. [94], who showed that chronic expression of IL- $1 \beta$ in adult rat $\mathrm{SNpc}$ using a recombinant adenovirus caused the death of dopaminergic neurons after three weeks. Mangano and Hayley [89] also suggested that the increase in inflammatory cytokines IL-6, IL-2, TNF- $\alpha$, and IFN- $\gamma$ was responsible for this effect. Villarán et al. [90] showed that the loss of TH-positive neurons induced in UC-LPS animals was produced along with a significant increase in microglial activation, and almost doubled that produced in animals treated with LPS alone. Moreover, it is worth noting that the authors found a significant increase in many of the cytokines studied in the animals with UC, such as TNF- $\alpha$, IL- $1 \beta$, and IL-6. This effect was increased in the UC-LPS animals. These results could be in agreement with Godoy et al. [19], especially regarding the increase in cytokines, in spite of their being unable to distinguish the effect of each in relationship to the degeneration of DAergic neurons. In this work, the authors also found a significant increase in inducible nitric oxide synthase (iNOS), which could also be involved in the effect of peripheral inflammation, which in turn could be in agreement with Cunningham et al. [83] who found that IL-1 could exacerbate disease symptoms in a prion disease model. Moreover, the relationship between these cytokines is worth noting. Koprich et al. [88] described that their model's suppression of LPS sensitivity, produced by the systemic treatment with IL-1ra, also produced a reduction in the levels of the proinflammatory cytokines IFN- $\gamma$ and TNF- $\alpha$ in the SN, supporting the cross-regulation between them, as had been previously pointed out. TNF$\alpha$ is also toxic to DA neurons when injected into the rat's brain, but the toxicity is greater when TNF- $\alpha$ and IL- $1 \beta$ were injected together [95]. A study using IL- $1 \beta$ and TNF$\alpha$-neutralizing antibodies showed that approximately $50 \%$ of the LPS-induced DA neuronal cell death in primary cultures of rat midbrain was mediated by the production of these two cytokines [96]. The implication of TNF- $\alpha$ signalling in the destruction of SN DA neurons in animal models of PD have also been reported $[18,21,36,97]$. Moreover, TNF- $\alpha$ contributed to the nigrostriatal neurodegeneration provoked by several DA insults [18, 21, 33, 34, 48, 98, 99]. Recently, De Lella Ezcurra et al. [100] have reported that chronic expression of low levels of TNF- $\alpha$ by adenoviral expression in the SN elicits progressive neurodegeneration, delayed motor symptoms and microglia/macrophage activation. All these data suggest that the increase in the inflammatory parameters in the periphery (blood) as a result of peripheral inflammation induced the increase in inflammation in $\mathrm{SN}$ and consequently the synergistic effect on the nigrostriatal dopaminergic system. The mechanism is not well known, but it is possible to suggest that at least some of these cytokines could enter. Circulating IL- $1 \beta$ may activate central neurons in a direct manner, especially in regions where BBB is deficient [101]. Furthermore, blood-borne IL- $1 \beta$ seems to stimulate the synthesis of prostaglandin E2 in central vessels, which can then diffuse into brain parenchyma to activate neurons in the SNC [102]. It should be also taken into account that the increase in BBB permeability, as well as its rupture, has been described as consequence of some peripheral inflammatory processes; so, disruption of the $\mathrm{BBB}$ has been described during TBS colitis [103, 104]. Reyes et al. [105] have shown that peripheral thermal injuries produced an increase in BBB permeability and rupture. In vitro, these effects have been proved to be produced by TNF- $\alpha$, IL- $1 \beta$, and IL- 6 through the COX pathway [106]. In vivo, upregulation of TNF- $\alpha$ and IL- $1 \beta$, as well as promoted blood-borne inflammatory cell adherence and infiltration, may be responsible [107-109]. Many studies have shown that cytokines may play an important role in the alteration of $\mathrm{BBB}$ function [109-112]. There is no agreement in the reports on rupture or increased permeability of the BBB in PD [113115]. However, PET and histological studies on PD patients have shown dysfunction in the BBB transporter system [69] and alteration of blood vessels [70] in the midbrain of PD patients. Additionally, the levels of VEGF and PEDF that induce structural changes in blood vessels increased in PD patients and the MPTP model [71]. Moreover, Rite et al. [72] showed disrupted BBB and induced DA neuronal death in the ventral mesencephalon after injecting VEGF within the $\mathrm{SN}$. All these data allow us to suggest that these circumstances could favour conditions in which peripheral cytokines were able to enter the CNS.

The degenerative processes of the nigrostriatal DAergic system in PD could be related to McGeer's description of a massive number of cytotoxic T cells ( Tc) in the SN of patients with PD [75], along with descriptions that the density of IFN- $\gamma$-positive cells was markedly higher in brains of patients with PD [116]. Both facts suggest that the recruitment of $T$ cells to the brain could be associated with the nigrostriatal pathway injury in PD. This possibility has been confirmed by Brochard et al. [77], who described that CD8+ and $\mathrm{CD} 4+\mathrm{T}$ cells, but not $\mathrm{B}$ cells, invaded the brain during the course of neuronal degeneration in the MPTP animal model of PD. The effect of these cells was reinforced by the fact that the MPTP-induced dopaminergic cell death was markedly attenuated in the absence of mature $\mathrm{T}$ lymphocytes in two different immunodeficient mice strains (Rag1-/and $T \mathrm{crb}-/-$ mice). However, there was no protection in mice lacking CD8. The implication of these cells was strongly supported, since the authors also found that both $\mathrm{CD} 8+$ and CD4+ T cells accumulated markedly in the SNpc of PD patients. These data indicate that $\mathrm{T}$ cell-mediated dopaminergic toxicity is almost exclusively mediated by CD4+ T cells. The authors also point out that the lymphocyte infiltration into the brain is not a passive phenomenon, suggesting that the activation of microglia along innate neuroinflammatory processes with the modification of the local microenvironment could be involved. They found the upregulated expression of the intercellular adhesion molecule-1 (ICAM-1) adhesion molecule on both capillaries 
and glial cells, which may participate in the attachment of leukocytes to the vascular endothelium and their diapedesis [117]. In this context, Villarán et al. [90] has shown the effect of peripheral inflammation (UC) on the infiltration of circulating monocytes in the SN using flow cytometry analyses. They found that monocytes infiltration into the $\mathrm{SN}$ increased in the UC-LPS animals compared with the animals treated with LPS alone. Moreover, they showed the reversion of most of the deleterious effects of peripheral inflammation on microglial activation, BBB disruption, astrocytes loss, and degeneration of nigral dopaminergic neurons induced by LPS after using clodronate encapsulated in liposomes (ClodLip), which produced a peripheral macrophage depletion lasting 5 days in blood, liver, and spleen of normal rats and mice [118-120]. Taken together, these results suggest that peripheral inflammation induced by UC contributes to dopaminergic degeneration; the activation of macrophages seems to play some role, since the destruction of this peripheral leukocyte type by ClodLip abolishes the damaging effects associated with UC in the ventral mesencephalon. Brochard et al. [77] suggested that the overexpression of the ICAM-1 adhesion molecule could be involved in the active migration of these cells. Villarán et al. [90] also found a significant increase in ICAM-1 in the SN of UC-LPS animals. It is also interesting to note that the overexpression of ICAM-1 was also described in the SN of patients with PD and in MPTP-intoxicated monkeys [121], supporting a role for immune regulation outside the CNS and the possible implication of peripheral inflammation.

All these data show that peripheral inflammation could enhance the degeneration of the nigrostriatal system, previously induced by an insult. Therefore, PD symptoms could appear earlier, increasing the incidence of the disease. An increased prevalence of peptic ulcer prodromal to idiopathic Parkinsonism has been described [81, 122]. This prompted some authors to suggest a prominent role of inflammation in the gastrointestinal tract in the aetiology and pathogenesis of idiopathic Parkinsonism, including a possible role for Helicobacter pylori infections $[82,123]$. This infective process is the most prevalent in the world, affecting approximately $50 \%$ of the population [124], and it is considered the causative agent of many gastrointestinal and extradigestive conditions. Colonization of gastric mucosa by $H$. pylori is accompanied by an inflammatory response associated with gastric mucosal damage through the activation of polymorphonuclear neutrophil leukocytes [125] and inflammatory infiltration of lymphocytes, plasma cells, and macrophages in the stomach tissue [126-128]. Also described was the production of proinflammatory factors such as IL-8, IL-1, and TNF- $\alpha[115,129,130]$.

It should not be ignored that a high peripheral inflammation could induce the degeneration of the nigrostriatal system alone and also that peripheral inflammation could produce a special sensitivity to other dopaminergic insults. An increase in PD was reported following the Spanish flu in 1918 [131]; however, the viral RNA is reported absent from brain samples of encephalitis lethargica patients from 1916 to 1920 [132]. It is conceivable that the massive immune response or "cytokine storm" [133] created by the virus initiated inflammation in the CNS; also, the great peripheral inflammation could have affected the nigrostriatal dopaminergic neurons. The inflammation produced by a single injection of a large dose of LPS into the periphery has been shown to produce inflammation in the brain, resulting in significant DA neuron loss in the SN [134]. Villarán et al. [90] also reported a decrease in TH positive neurons in the SN in animals with UC after injecting vehicle within this structure. The specific sensitization was reported by Ling et al. [135], who showed that prenatal exposure to LPS increased DA cell loss following adult exposure to 6-OHDA.

\section{Conclusions}

Our aim was to point out that inflammation is able to enhance the damage to DAergic neurons and, more importantly, that peripheral inflammation is also able to produce a synergistic increase in the effect produced by any insult in the nigrostriatal dopaminergic system, resulting in a greater loss of DA neurons. Consequently, this effect increases the progression rate of PD. The effect of peripheral inflammation seems to be produced through a significantly increased inflammation in the SN. This could be produced by some of the cytokines that increase in blood, such as IL- $\beta$ and TNF$\alpha$, directly or indirectly through its transport to the CNS $(\mathrm{SN})$. Moreover, these effects could be also accompanied by the recruitment of peripheral monocytes that also directly increase the inflammation process in the SN. The implication of peripheral inflammation could explain some epidemiological data on the incidence of $\mathrm{PD}$, and probably also the effect of chronic anti-inflammatory treatments. These effects should be taken into account in the progression of PD.

\section{Acknowledgments}

This work was supported by Grant no. SAF2008-04366. M. Sarmiento thanks the Junta de Andalucía for a Beca de Proyecto de Excelencia grant; M. J. Delgado-Cortés and R. Mauriño thank the Ministerio de Educación for their FPU and FPI fellowships, respectively. The authors thank Mr. Mark Elliott for the revision of the paper.

\section{References}

[1] J. Poirier, S. Kogan, and S. Gauthier, "Environment, genetics and idiopathic Parkinson's disease," Canadian Journal of Neurological Sciences, vol. 18, no. 1, pp. 70-76, 1991.

[2] F. Tsang and T. W. Soong, "Interactions between environmental and genetic factors in the pathophysiology of Parkinson's disease," IUBMB Life, vol. 55, no. 6, pp. 323-327, 2003.

[3] M. F. Allam, A. S. Del Castillo, and R. F. Navajas, "Parkinson's disease risk factors: genetic, environmental, or both?" Neurological Research, vol. 27, no. 2, pp. 206-208, 2005.

[4] Y. C. Chung, H. W. Ko, E. Bok et al., "The role of neuroinflammation on the pathogenesis of Parkinson's disease," BMB Reports, vol. 43, no. 4, pp. 225-232, 2010. 
[5] P. L. McGeer, S. Itagaki, B. E. Boyes, and E. G. McGeer, "Reactive microglia are positive for HLA-DR in the substantia nigra of Parkinson's and Alzheimer's disease brains," Neurology, vol. 38, no. 8, pp. 1285-1291, 1988.

[6] E. C. Hirsch, S. Hunot, P. Damier, and B. Faucheux, "Glial cells and inflammation in Parkinson's disease: a role in neurodegeneration?" Annals of Neurology, vol. 44, no. 3, supplement 1, pp. S115-S120, 1998.

[7] P. S. Whitton, "Inflammation as a causative factor in the aetiology of Parkinson's disease," British Journal of Pharmacology, vol. 150, no. 8, pp. 963-976, 2007.

[8] P. L. McGeer and E. G. McGeer, "The inflammatory response system of brain: implications for therapy of Alzheimer and other neurodegenerative diseases," Brain Research Reviews, vol. 21, no. 2, pp. 195-218, 1995.

[9] E. C. Hirsch, S. Hunot, B. Faucheux et al., "Dopaminergic neurons degenerate by apoptosis in Parkinson's disease," Movement Disorders, vol. 14, no. 2, pp. 383-384, 1999.

[10] M. Mogi and T. Nagatsu, "Neurotrophins and cytokines in Parkinson's disease," Advances in Neurology, vol. 80, pp. 135139, 1999.

[11] A. J. Herrera, M. Tomás-Camardiel, J. L. Venero, J. Cano, and A. Machado, "Inflammatory process as a determinant factor for the degeneration of substantia nigra dopaminergic neurons," Journal of Neural Transmission, vol. 112, no. 1, pp. 111-119, 2005.

[12] T. Nagatsu and M. Sawada, "Inflammatory process in Parkinson's disease: role for cytokines," Current Pharmaceutical Design, vol. 11, no. 8, pp. 999-1016, 2005.

[13] S. Hunot, F. Boissière, B. Faucheux et al., "Nitric oxide synthase and neuronal vulnerability in Parkinson's disease," Neuroscience, vol. 72, no. 2, pp. 355-363, 1996.

[14] C. Knott, G. Stern, and G. P. Wilkin, "Inflammatory regulators in Parkinson's disease: iNOS, lipocortin-1, and cyclooxygenases-1 and -2," Molecular and Cellular Neuroscience, vol. 16, no. 6, pp. 724-739, 2000.

[15] W. D. Le, D. B. Rowe, J. Jankovic, W. Xie, and S. H. Appel, "Effects of cerebrospinal fluid from patients with Parkinson disease on dopaminergic cells," Archives of Neurology, vol. 56, no. 2, pp. 194-200, 1999.

[16] M. Mogi, M. Harada, P. Riederer, H. Narabayashi, K. Fujita, and T. Nagatsu, "Tumor necrosis factor- $\alpha$ (TNF- $\alpha$ ) increases both in the brain and in the cerebrospinal fluid from parkinsonian patients," Neuroscience Letters, vol. 165, no. 1-2, pp. 208-210, 1994.

[17] M. Mogi, M. Harada, H. Narabayashi, H. Inagaki, M. Minami, and T. Nagatsu, "Interleukin (IL)-1 $\beta$, IL-2, IL-4, IL- 6 and transforming growth factor- $\alpha$ levels are elevated in ventricular cerebrospinal fluid in juvenile parkinsonism and Parkinson's disease," Neuroscience Letters, vol. 211, no. 1, pp. 13-16, 1996.

[18] B. Ferger, A. Leng, A. Mura, B. Hengerer, and J. Feldon, "Genetic ablation of tumor necrosis factor-alpha (TNF- $\alpha$ ) and pharmacological inhibition of TNF-synthesis attenuates MPTP toxicity in mouse striatum," Journal of Neurochemistry, vol. 89, no. 4, pp. 822-833, 2004.

[19] M. C. P. Godoy, R. Tarelli, C. C. Ferrari, M. I. Sarchi, and F. J. Pitossi, "Central and systemic IL-1 exacerbates neuro degeneration and motor symptoms in a model of Parkinson's disease," Brain, vol. 131, no. 7, pp. 1880-1894, 2008.

[20] P. Klevenyi, O. Andreassen, R. J. Ferrante, J. R. Schleicher, R. M. Friedlander, and M. F. Beal, "Transgenic mice expressing a dominant negative mutant interleukin- $1 \beta$ converting enzyme show resistance to MPTP neurotoxicity," NeuroReport, vol. 10, no. 3, pp. 635-638, 1999.

[21] K. Sriram, J. M. Matheson, S. A. Benkovic, D. B. Miller, M. I. Luster, and J. P. O'Callaghan, "Mice deficient in TNF receptors are protected against dopaminergic neurotoxicity: implications for Parkinson's disease," The FASEB Journal, vol. 16, no. 11, pp. 1474-1476, 2002.

[22] G. Banisadr, W. Rostène, P. Kitabgi, and S. M. Parsadaniantz, "Chemokines and brain functions," Current Drug Targets: Inflammation and Allergy, vol. 4, no. 3, pp. 387-399, 2005.

[23] L. Cartier, O. Hartley, M. Dubois-Dauphin, and K. H. Krause, "Chemokine receptors in the central nervous system: role in brain inflammation and neurodegenerative diseases," Brain Research Reviews, vol. 48, no. 1, pp. 16-42, 2005.

[24] K. Biber, E. K. de Jong, H. R. J. van Weering, and H. W. G. M. Boddeke, "Chemokines and their receptors in central nervous system disease," Current Drug Targets, vol. 7, no. 1, pp. 29-46, 2006.

[25] M. Minami, T. Katayama, and M. Satoh, "Brain cytokines and chemokines: roles in ischemic injury and pain," Journal of Pharmacological Sciences, vol. 100, no. 5, pp. 461-470, 2006.

[26] M. Nishimura, I. Mizuta, E. Mizuta, S. Yamasaki, M. Ohta, and S. Kuno, "Influence of interleukin- $1 \beta$ gene polymorphisms on age-at-onset of sporadic Parkinson's disease," Neuroscience Letters, vol. 284, no. 1-2, pp. 73-76, 2000.

[27] T. Schulte, L. Schöls, T. Müller, D. Woitalla, K. Berger, and R. Krüger, "Polymorphisms in the interleukin-1 alpha and beta genes and the risk for Parkinson's disease," Neuroscience Letters, vol. 326, no. 1, pp. 70-72, 2002.

[28] M. Nishimura, S. Kuno, I. Mizuta et al., "Influence of monocyte chemoattractant protein 1 gene polymorphism on age at onset of sporadic Parkinson's disease," Movement Disorders, vol. 18, no. 8, pp. 953-955, 2003.

[29] C. Huerta, V. Álvarez, I. F. Mata et al., "Chemokines (RANTES and MCP-1) and chemokine-receptors (CCR2 and CCR5) gene polymorphisms in Alzheimer's and Parkinson's disease," Neuroscience Letters, vol. 370, no. 2-3, pp. 151-154, 2004.

[30] O. A. Ross, C. O’Neill, I. M. Rea et al., "Functional promoter region polymorphism of the proinflammatory chemokine IL-8 gene associates with Parkinson's disease in the Irish," Human Immunology, vol. 65, no. 4, pp. 340-346, 2004.

[31] A. Håkansson, L. Westberg, S. Nilsson et al., "Interaction of polymorphisms in the genes encoding interleukin- 6 and estrogen receptor beta on the susceptibility to Parkinson's disease," American Journal of Medical Genetics, vol. 133, no. 1, pp. 88-92, 2005.

[32] R. Pattarini, R. J. Smeyne, and J. I. Morgan, "Temporal mRNA profiles of inflammatory mediators in the murine 1-methyl-4-phenyl-1,2,3,6-tetrahydropyrimidine model of Parkinson's disease," Neuroscience, vol. 145, no. 2, pp. 654668, 2007.

[33] K. Sriram, J. M. Matheson, S. A. Benkovic, D. B. Miller, M. I. Luster, and J. P. O'Callaghan, "Deficiency of TNF receptors suppresses microglial activation and alters the susceptibility of brain regions to MPTP-induced neurotoxicity: role of TNF- $\alpha$," The FASEB Journal, vol. 20, no. 6, pp. 670-682, 2006.

[34] K. Sriram, D. B. Miller, and J. P. O'Callaghan, "Minocycline attenuates microglial activation but fails to mitigate striatal dopaminergic neurotoxicity: role of tumor necrosis factor$\alpha$," Journal of Neurochemistry, vol. 96, no. 3, pp. 706-718, 2006. 
[35] E. Rousselet, J. Callebert, K. Parain et al., "Role of TNF- $\alpha$ receptors in mice intoxicated with the parkinsonian toxin MPTP," Experimental Neurology, vol. 177, no. 1, pp. 183-192, 2002.

[36] A. Leng, A. Mura, J. Feldon, and B. Ferger, "Tumor necrosis factor-alpha receptor ablation in a chronic MPTP mouse model of Parkinson's disease," Neuroscience Letters, vol. 375, no. 2, pp. 107-111, 2005.

[37] L. M. Bolin, I. Strycharska-Orczyk, R. Murray, J. W. Langston, and D. Di Monte, "Increased vulnerability of dopaminergic neurons in MPTP-lesioned interleukin-6 deficient mice," Journal of Neurochemistry, vol. 83, no. 1, pp. 167$175,2002$.

[38] Z. Liu, Y.-H. Qiu, B. Li, S.-H. Ma, and Y.-P. Peng, "Neuroprotection of interleukin-6 against NMDA-induced apoptosis and its signal-transduction mechanisms," Neurotoxicity Research, vol. 19, no. 3, pp. 484-495, 2011.

[39] H. Chen, S. M. Zhang, M. A. Hernán et al., "Nonsteroidal anti-inflammatory drugs and the risk of Parkinson disease," Archives of Neurology, vol. 60, no. 8, pp. 1059-1064, 2003.

[40] H. Chen, E. Jacobs, M. A. Schwarzschild et al., "Nonsteroidal antiinflammatory drug use and the risk for Parkinson's disease," Annals of Neurology, vol. 58, no. 6, pp. 963-967, 2005.

[41] M. Schiess, "Nonsteroidal anti-inflammatory drugs protect against Parkinson neurodegeneration: can an NSAID a day keep Parkinson disease away?" Archives of Neurology, vol. 60, no. 8, pp. 1043-1044, 2003.

[42] M. A. Hernán, G. Logroscino, and L. A. García Rodríguez, "Nonsteroidal anti-inflammatory drugs and the incidence of Parkinson disease," Neurology, vol. 66, no. 7, pp. 1097-1099, 2006.

[43] D. B. Hancock, E. R. Martin, J. M. Stajich et al., "Smoking, caffeine, and nonsteroidal anti-inflammatory drugs in families with Parkinson disease," Archives of Neurology, vol. 64, no. 4, pp. 576-580, 2007.

[44] T. G. Ton, S. R. Heckbert, W. T. Longstreth et al., "Nonsteroidal anti-inflammatory drugs and risk of Parkinson's disease," Movement Disorders, vol. 21, no. 7, pp. 964-969, 2006.

[45] B. Ferger, P. Teismann, C. D. Earl, K. Kuschinsky, and W. H. Oertel, "Salicylate protects against MPTP-induced impairments in dopaminergic neurotransmission at the striatal and nigral level in mice," Naunyn-Schmiedeberg's Archives of Pharmacology, vol. 360, no. 3, pp. 256-261, 1999.

[46] K. Sairam, K. S. Saravanan, R. Banerjee, and K. P. Mohanakumar, "Non-steroidal anti-inflammatory drug sodium salicylate, but not diclofenac or celecoxib, protects against 1methyl-4-phenyl pyridinium-induced dopaminergic neurotoxicity in rats," Brain Research, vol. 966, no. 2, pp. 245-252, 2003.

[47] D. S. Maharaj, K. S. Saravanan, H. Maharaj, K. P. Mohanakumar, and S. Daya, "Acetaminophen and aspirin inhibit superoxide anion generation and lipid peroxidation, and protect against 1-methyl-4-phenyl pyridinium-induced dopaminergic neurotoxicity in rats," Neurochemistry International, vol. 44, no. 5, pp. 355-360, 2004.

[48] D. C. Wu, V. Jackson-Lewis, M. Vila et al., "Blockade of microglial activation is neuroprotective in the 1-methyl-4phenyl-1,2,3,6-tetrahydropyridine mouse model of Parkinson disease," The Journal of Neuroscience, vol. 22, no. 5, pp. 1763-1771, 2002.
[49] A. Castaño, A. J. Herrera, J. Cano, and A. Machado, "Lipopolysaccharide intranigral injection induces inflammatory reaction and damage in nigrostriatal dopaminergic system," Journal of Neurochemistry, vol. 70, no. 4, pp. 15841592, 1998.

[50] A. Castaño, A. J. Herrera, J. Cano, and A. Machado, "The degenerative effect of a single intranigral injection of LPS on the dopaminergic system is prevented by dexamethasone, and not mimicked by rh-TNF- $\alpha$ IL- $1 \beta$ IFN- $\gamma$," Journal of Neurochemistry, vol. 81, no. 1, pp. 150-157, 2002.

[51] A. J. Herrera, A. Castaño, J. L. Venero, J. Cano, and A. Machado, "The single intranigral injection of LPS as a new model for studying the selective effects of inflammatory reactions on dopaminergic system," Neurobiology of Disease, vol. 7, no. 4, pp. 429-447, 2000.

[52] A. J. Herrera, R. M. De Pablos, E. Carreño-Müller et al., "The intrastriatal injection of thrombin in rat induced a retrograde apoptotic degeneration of nigral dopaminergic neurons through synaptic elimination," Journal of Neurochemistry, vol. 105, no. 3, pp. 750-762, 2008.

[53] E. Carreño-Müller, A. J. Herrera, R. M. de Pablos et al., "Thrombin induces in vivo degeneration of nigral dopaminergic neurones along with the activation of microglia," Journal of Neurochemistry, vol. 84, no. 5, pp. 1201-1214, 2003.

[54] W. G. Kim, R. P. Mohney, B. Wilson, G. H. Jeohn, B. Liu, and J. S. Hong, "Regional difference in susceptibility to lipopolysaccharide-induced neurotoxicity in the rat brain: role of microglia," The Journal of Neuroscience, vol. 20, no. 16, pp. 6309-6316, 2000.

[55] R. M. De Pablos, A. J. Herrera, R. F. Villarán, J. Cano, and A. Machado, "Dopamine-dependent neurotoxicity of lipopolysaccharide in substantia nigra," The FASEB Journal, vol. 19, no. 3, pp. 407-409, 2005.

[56] R. M. de Pablos, R. F. Villarán, S. Argüelles et al., "Stress increases vulnerability to inflammation in the rat prefrontal cortex," The Journal of Neuroscience, vol. 26, no. 21, pp. 57095719, 2006.

[57] M. Tomás-Camardiel, I. Rite, A. J. Herrera et al., "Minocycline reduces the lipopolysaccharide-induced inflammatory reaction, peroxynitrite-mediated nitration of proteins, disruption of the blood-brain barrier, and damage in the nigral dopaminergic system," Neurobiology of Disease, vol. 16, no. 1, pp. 190-201, 2004.

[58] M. D. C. Hernández-Romero, S. Argüelles, R. F. Villarán et al., "Simvastatin prevents the inflammatory process and the dopaminergic degeneration induced by the intranigral injection of lipopolysaccharide," Journal of Neurochemistry, vol. 105, no. 2, pp. 445-459, 2008.

[59] S. Argüelles, A. J. Herrera, E. Carreño-Müller et al., "Degeneration of dopaminergic neurons induced by thrombin injection in the substantia nigra of the rat is enhanced by dexamethasone: role of monoamine oxidase enzyme," NeuroToxicology, vol. 31, no. 1, pp. 55-66, 2010.

[60] R. F. Villarán, R. M. de Pablos, S. Argüelles et al., “The intranigral injection of tissue plasminogen activator induced blood-brain barrier disruption, inflammatory process and degeneration of the dopaminergic system of the rat," NeuroToxicology, vol. 30, no. 3, pp. 403-413, 2009.

[61] M. Critchley, "Medical aspects of boxing, particularly from a neurological standpoint," British Medical Journal, vol. 1, no. 5015, pp. 357-362, 1957. 
[62] A. H. Roberts, Brain Damage in Boxers. A Study of Prevalence of Traumatic Encephalopathy among Ex-Professional Boxers, Pitman Medical, London, UK, 1969.

[63] I. R. Casson, O. Siegel, and R. Sham, "Brain damage in modern boxers," Journal of the American Medical Association, vol. 251, no. 20, pp. 2663-2667, 1984.

[64] A. Guterman and R. W. Smith, "Neurological sequelae of boxing," Sports Medicine, vol. 4, no. 3, pp. 194-210, 1987.

[65] J. H. Friedman, "Progressive parkinsonism in boxers," Southern Medical Journal, vol. 82, no. 5, pp. 543-546, 1989.

[66] H. Clausen, P. McCrory, and V. Anderson, "The risk of chronic traumatic brain injury in professional boxing: change in exposure variables over the past century," British Journal of Sports Medicine, vol. 39, no. 9, pp. 661-664, 2005.

[67] D. J. Loane and K. R. Byrnes, "Role of microglia in neurotrauma," Neurotherapeutics, vol. 7, no. 4, pp. 366-377, 2010.

[68] B. O. Popescu, E. C. Toescu, L. M. Popescu et al., "Bloodbrain barrier alterations in ageing and dementia," Journal of the Neurological Sciences, vol. 283, no. 1-2, pp. 99-106, 2009.

[69] R. Kortekaas, K. L. Leenders, J. C. H. van Oostrom et al., "Blood-brain barrier dysfunction in Parkinsonian midbrain in vivo," Annals of Neurology, vol. 57, no. 2, pp. 176-179, 2005.

[70] B. A. Faucheux, A. M. Bonnet, Y. Agid, and E. C. Hirsch, "Blood vessels change in the mesencephalon of patients with Parkinson's disease," The Lancet, vol. 353, no. 9157, pp. 981982, 1999.

[71] T. Yasuda, M. Fukuda-Tani, T. Nihira et al., "Correlation between levels of pigment epithelium-derived factor and vascular endothelial growth factor in the striatum of patients with Parkinson's disease," Experimental Neurology, vol. 206, no. 2, pp. 308-317, 2007.

[72] I. Rite, A. Machado, J. Cano, and J. L. Venero, "Blood-brain barrier disruption induces in vivo degeneration of nigral dopaminergic neurons," Journal of Neurochemistry, vol. 101, no. 6, pp. 1567-1582, 2007.

[73] X. Chen, X. Lan, I. Roche, R. Liu, and J. D. Geiger, "Caffeine protects against MPTP-induced blood-brain barrier dysfunction in mouse striatum," Journal of Neurochemistry, vol. 107, no. 4, pp. 1147-1157, 2008.

[74] E. Yan, M. Castillo-Meléndez, T. Nicholls, J. Hirst, and D. Walker, "Cerebrovascular responses in the fetal sheep brain to low-dose endotoxin," Pediatric Research, vol. 55, no. 5, pp. 855-863, 2004.

[75] P. L. McGeer, S. Itagaki, H. Akiyama, and E. G. McGeer, "Rate of cell death in parkinsonism indicates active neuropathological process," Annals of Neurology, vol. 24, no. 4, pp. 574-576, 1988.

[76] K. A. Ji, M. S. Yang, H. K. Jeong et al., "Resident microglia die and infiltrated neutrophils and monocytes become major inflammatory cells in lipopolysaccharide-injected brain," Glia, vol. 55, no. 15, pp. 1577-1588, 2007.

[77] V. Brochard, B. Combadière, A. Prigent et al., "Infiltration of $\mathrm{CD} 4{ }^{+}$lymphocytes into the brain contributes to neurodegeneration in a mouse model of Parkinson disease," The Journal of Clinical Investigation, vol. 119, no. 1, pp. 182-192, 2009.

[78] E. Esposito, V. Di Matteo, A. Benigno, M. Pierucci, G. Crescimanno, and G. Di Giovanni, "Non-steroidal antiinflammatory drugs in Parkinson's disease," Experimental Neurology, vol. 205, no. 2, pp. 295-312, 2007.
[79] R. S. Tilvis, M. H. Kähönen-Väre, J. Jolkkonen, J. Valvanne, K. H. Pitkala, and T. E. Strandberg, "Predictors of cognitive decline and mortality of aged people over a 10-year period," The Journals of Gerontology Series A, vol. 59, no. 3, pp. 268274, 2004.

[80] S. Serres, D. C. Anthony, Y. Jiang et al., "Systemic inflammatory response reactivates immune-mediated lesions in rat brain," The Journal of Neuroscience, vol. 29, no. 15, pp. 4820$4828,2009$.

[81] R. R. Strang, "The association of gastro-duodenal ulceration with Parkinson's disease," The Medical Journal of Australia, vol. 310, pp. 842-843, 1965.

[82] R. J. Dobbs, S. M. Dobbs, C. Weller et al., "Role of chronic infection and inflammation in the gastrointestinal tract in the etiology and pathogenesis of idiopathic parkinsonism. Part 1: eradication of Helicobacter in the cachexia of idiopathic parkinsonism," Helicobacter, vol. 10, no. 4, pp. 267-275, 2005.

[83] C. Cunningham, D. C. Wilcockson, S. Campion, K. Lunnon, and V. H. Perry, "Central and systemic endotoxin challenges exacerbate the local inflammatory response and increase neuronal death during chronic neurodegeneration," The Journal of Neuroscience, vol. 25, no. 40, pp. 9275-9284, 2005.

[84] H. M. Gao, J. S. Hong, W. Zhang, and B. Liu, "Synergistic dopaminergic neurotoxicity of the pesticide rotenone and inflammogen lipopolysaccharide: relevance to the etiology of Parkinson's disease," The Journal of Neuroscience, vol. 23, no. 4, pp. 1228-1236, 2003.

[85] H.-M. Gao, B. Liu, W. Zhang, and J.-S. Hong, "Synergistic dopaminergic neurotoxicity of MPTP and inflammogen lipopolysaccharide: relevance to the etiology of Parkinson's disease," The FASEB Journal, vol. 17, no. 13, pp. 1957-1959, 2003.

[86] C. M. Long-Smith, L. Collins, A. Toulouse, A. M. Sullivan, and Y. M. Nolan, "Interleukin- $1 \beta$ contributes to dopaminergic neuronal death induced by lipopolysaccharide-stimulated rat glia in vitro," Journal of Neuroimmunology, vol. 226, no. 12, pp. 20-26, 2010.

[87] P. Zhang, K. M. Lokuta, D. E. Turner, and B. Liu, "Synergistic dopaminergic neurotoxicity of manganese and lipopolysaccharide: differential involvement of microglia and astroglia," Journal of Neurochemistry, vol. 112, no. 2, pp. 434-443, 2010.

[88] J. B. Koprich, C. Reske-Nielsen, P. Mithal, and O. Isacson, "Neuroinflammation mediated by IL- $1 \beta$ increases susceptibility of dopamine neurons to degeneration in an animal model of Parkinson's disease," Journal of Neuroinflammation, vol. 5, article 8, 2008.

[89] E. N. Mangano and S. Hayley, "Inflammatory priming of the substantia nigra influences the impact of later paraquat exposure: neuroimmune sensitization of neurodegeneration," Neurobiology of Aging, vol. 30, no. 9, pp. 1361-1378, 2009.

[90] R. F. Villarán, A. M. Espinosa-Oliva, M. Sarmiento et al., "Ulcerative colitis exacerbates lipopolysaccharide-induced damage to the nigral dopaminergic system: potential risk factor in Parkinson's disease," Journal of Neurochemistry, vol. 114, no. 6, pp. 1687-1700, 2010.

[91] I. Okayasu, S. Hatakeyama, M. Yamada, T. Ohkusa, Y. Inagaki, and R. Nakaya, "A novel method in the induction of reliable experimental acute and chronic ulcerative colitis in mice," Gastroenterology, vol. 98, no. 3, pp. 694-702, 1990.

[92] E. Gaudio, G. Taddei, A. Vetuschi et al., "Dextran sulfate sodium (DSS) colitis in rats: clinical, structural, and ultrastructural aspects," Digestive Diseases and Sciences, vol. 44, no. 7, pp. 1458-1475, 1999. 
[93] M. C. Pott Godoy, C. C. Ferrari, and F. J. Pitossi, "Nigral neurodegeneration triggered by striatal AdIL-1 administration can be exacerbated by systemic IL-1 expression," Journal of Neuroimmunology, vol. 222, no. 1-2, pp. 29-39, 2010.

[94] C. C. Ferrari, M. C. Pott Godoy, R. Tarelli, M. Chertoff, A. M. Depino, and F. J. Pitossi, "Progressive neurodegeneration and motor disabilities induced by chronic expression of IL- $1 \beta$ in the substantia nigra," Neurobiology of Disease, vol. 24, no. 1, pp. 183-193, 2006.

[95] P. M. Carvey, E. Y. Chen, J. W. Lipton, C. W. Tong, Q. A. Chang, and Z. D. Ling, "Intra-parenchymal injection of tumor necrosis factor- $\alpha$ and interleukin $1-\beta$ produces dopamine neuron loss in the rat," Journal of Neural Transmission, vol. 112, no. 5, pp. 601-612, 2005.

[96] D. A. Gayle, Z. Ling, C. Tong, T. Landers, J. W. Lipton, and P. M. Carvey, "Lipopolysaccharide (LPS)-induced dopamine cell loss in culture: roles of tumor necrosis factor$\alpha$, interleukin- $1 \beta$, and nitric oxide," Brain Research. Developmental Brain Research, vol. 133, no. 1, pp. 27-35, 2002.

[97] M. K. McCoy, T. N. Martinez, K. A. Ruhn et al., "Blocking soluble tumor necrosis factor signaling with dominantnegative tumor necrosis factor inhibitor attenuates loss of dopaminergic neurons in models of Parkinson's disease," The Journal of Neuroscience, vol. 26, no. 37, pp. 9365-9375, 2006.

[98] D. Y. Lee, Y. J. Oh, and B. K. Jin, "Thrombin-activated microglia contribute to death of dopaminergic neurons in rat mesencephalic cultures: dual roles of mitogen-activated protein kinase signaling pathways," Glia, vol. 51, no. 2, pp. 98-110, 2005.

[99] H. Takeuchi, S. Jin, J. Wang et al., "Tumor necrosis factor$\alpha$ induces neurotoxicity via glutamate release from hemichannels of activated microglia in an autocrine manner," The Journal of Biological Chemistry, vol. 281, no. 30, pp. 21362-21368, 2006.

[100] A. L. De Lella Ezcurra, M. Chertoff, C. Ferrari, M. Graciarena, and F. Pitossi, "Chronic expression of low levels of tumor necrosis factor- $\alpha$ in the substantia nigra elicits progressive neurodegeneration, delayed motor symptoms and microglia/macrophage activation," Neurobiology of Disease, vol. 37, no. 3, pp. 630-640, 2010.

[101] A. M. van Dam, J. G. J. M. Bol, R. P. A. Gaykema et al., "Vagotomy does not inhibit high dose lipopolysaccharideinduced interleukin- $1 \beta$ immunoreactivity in rat brain and pituitary gland," Neuroscience Letters, vol. 285, no. 3, pp. 169-172, 2000.

[102] M. Ek, D. Engblom, S. Saha, A. Blomqvist, P.-J. Jakobsson, and A. Ericsson-Dahlstrand, "Pathway across the bloodbrain barrier," Nature, vol. 410, no. 6827, pp. 430-431, 2001.

[103] C. A. Hathaway, C. B. Appleyard, W. H. Percy, and J. L. Williams, "Experimental colitis increases blood-brain barrier permeability in rabbits," American Journal of Physiology, vol. 276, no. 5, part 1, pp. G1174-G1180, 1999.

[104] S. S. Natah, A. Mouihate, Q. J. Pittman, and K. A. Sharkey, "Disruption of the blood-brain barrier during TNBS colitis," Neurogastroenterology and Motility, vol. 17, no. 3, pp. 433-446, 2005.

[105] R. Reyes Jr., Y. Wu, Q. Lai et al., "Early inflammatory response in rat brain after peripheral thermal injury," Neuroscience Letters, vol. 407, no. 1, pp. 11-15, 2006.

[106] H. E. De Vries, M. C. M. Blom-Roosemalen, M. van Oosten et al., "The influence of cytokines on the integrity of the blood-brain barrier in vitro," Journal of Neuroimmunology, vol. 64, no. 1, pp. 37-43, 1996.
[107] D. Stanimirovic and K. Satoh, "Inflammatory mediators of cerebral endothelium: a role in ischemic brain inflammation," Brain Pathology, vol. 10, no. 1, pp. 113-126, 2000.

[108] M. A. Petty and J. G. Wettstein, "Elements of cerebral microvascular ischaemia," Brain Research Reviews, vol. 36, no. 1, pp. 23-34, 2001.

[109] J. B. Dietrich, "The adhesion molecule ICAM-1 and its regulation in relation with the blood-brain barrier," Journal of Neuroimmunology, vol. 128, no. 1-2, pp. 58-68, 2002.

[110] J. E. Merrill and S. P. Murphy, "Inflammatory events at the blood brain barrier: regulation of adhesion molecules, cytokines, and chemokines by reactive nitrogen and oxygen species," Brain, Behavior, and Immunity, vol. 11, no. 4, pp. 245-263, 1997.

[111] N. J. Abbott, "Inflammatory mediators and modulation of blood-brain barrier permeability," Cellular and Molecular Neurobiology, vol. 20, no. 2, pp. 131-147, 2000.

[112] P. F. Stahel, E. Shohami, F. M. Younis et al., "Experimental closed head injury: analysis of neurological outcome, blood-brain barrier dysfunction, intracranial neutrophil infiltration, and neuronal cell death in mice deficient in genes for pro-inflammatory cytokines," Journal of Cerebral Blood Flow and Metabolism, vol. 20, no. 2, pp. 369-380, 2000.

[113] E. Farkas, G. I. de Jong, R. A. I. de Vos, E. N. H. Jansen Steur, and P. G. M. Luiten, "Pathological features of cerebral cortical capillaries are doubled in Alzheimer's disease and Parkinson's disease," Acta Neuropathologica, vol. 100, no. 4, pp. 395-402, 2000.

[114] J. D. Adams Jr., L. K. Klaidman, I. N. Odunze, and J. N. Johannessen, "Effects of MPTP on the cerebrovasculature," International Journal of Developmental Neuroscience, vol. 9, no. 2, pp. 155-159, 1991.

[115] C. Zhao, Z. Ling, M. B. Newman, A. Bhatia, and P. M. Carvey, "TNF- $\alpha$ knockout and minocycline treatment attenuates blood-brain barrier leakage in MPTP-treated mice," Neurobiology of Disease, vol. 26, no. 1, pp. 36-46, 2007.

[116] S. Hunot, N. Dugas, B. Faucheux et al., "FceRII/CD23 is expressed in Parkinson's disease and induces, in vitro, production of nitric oxide and tumor necrosis factor- $\alpha$ in glial cells," The Journal of Neuroscience, vol. 19, no. 9, pp. 3440-3447, 1999.

[117] T. A. Springer, "Traffic signals for lymphocyte recirculation and leukocyte emigration: the multistep paradigm," Cell, vol. 76, no. 2, pp. 301-314, 1994.

[118] N. van Rooijen, "The liposome-mediated macrophage 'suicide' technique," Journal of Immunological Methods, vol. 124, no. 1, pp. 1-6, 1989.

[119] N. van Rooijen, A. Sanders, and T. K. van den Berg, "Apoptosis of macrophages induced by liposome-mediated intracellular delivery of clodronate and propamidine," Journal of Immunological Methods, vol. 193, no. 1, pp. 93-99, 1996.

[120] G. A. P. Nieuwenhuijzen, M. F. C. M. Knapen, T. Hendriks, N. van Rooijen, and R. J. A. Goris, "Elimination of various subpopulations of macrophages and the development of multiple-organ dysfunction syndrome in mice," Archives of Surgery, vol. 132, no. 5, pp. 533-539, 1997.

[121] J. Miklossy, D. D. Doudet, C. Schwab, S. Yu, E. G. McGeer, and P. L. McGeer, "Role of ICAM-1 in persisting inflammation in Parkinson disease and MPTP monkeys," Experimental Neurology, vol. 197, no. 2, pp. 275-283, 2006.

[122] J. R. Warren and B. Marshall, "Unidentified curved bacilli on gastric epithelium in active chronic gastritis," The Lancet, vol. 1, no. 8336, pp. 1273-1275, 1983. 
[123] C. Weller, N. Oxlade, S. M. Dobbs, R. J. Dobbs, A. Charlett, and I. T. Bjarnason, "Role of inflammation in gastrointestinal tract in aetiology and pathogenesis of idiopathic parkinsonism," FEMS Immunology and Medical Microbiology, vol. 44, no. 2, pp. 129-135, 2005.

[124] F. Megraud, "Epidemiology of Helicobacter pylori infection," Gastroenterology Clinics of North America, vol. 22, no. 1, pp. 73-88, 1993.

[125] P. Ernst, "Review article: the role of inflammation in the pathogenesis of gastric cancer," Alimentary Pharmacology \& Therapeutics, vol. 13, supplement 1, pp. 13-18, 1999.

[126] T. Shimada and A. Terano, "Chemokine expression in Helicobacter pylori-infected gastric mucosa," Journal of Gastroenterology, vol. 33, no. 5, pp. 613-617, 1998.

[127] R. M. Peek, "Helicobacter pylori strain-specific activation of signal transduction cascades related to gastric inflammation," American Journal of Physiology, vol. 280, no. 4, pp. G525G530, 2001.

[128] S. Aydemir, I. O. Tekin, G. Numanoglu, A. Borazan, and Y. Ustundag, "Eosinophil infiltration, gastric juice and serum eosinophil cationic protein levels in Helicobacter pyloriassociated chronic gastritis and gastric ulcer," Mediators of Inflammation, vol. 13, no. 5-6, pp. 369-372, 2004.

[129] V. Supajatura, H. Ushio, A. Wada et al., "Cutting edge: VacA, a vacuolating cytotoxin of Helicobacter pylori, directly activates mast cells for migration and production of proinflammatory cytokines," Journal of Immunology, vol. 168, no. 6, pp. 2603-2607, 2002.

[130] S. Brandt, T. Kwok, R. Hartig, W. König, and S. Backert, "NF- $\kappa \mathrm{B}$ activation and potentiation of proinflammatory responses by the Helicobacter pylori CagA protein," Proceedings of the National Academy of Sciences of the United States of America, vol. 102, no. 26, pp. 9300-9305, 2005.

[131] R. T. Ravenholt and W. H. Foege, "1918 influenza, encephalitis lethargica, parkinsonism," The Lancet, vol. 2, no. 8303, pp. 860-864, 1982.

[132] K. C. Lo, J. F. Geddes, R. S. Daniels, and J. S. Oxford, "Lack of detection of influenza genes in archived formalin-fixed, paraffin wax-embedded brain samples of encephalitis lethargica patients from 1916 to 1920," Virchows Archiv, vol. 442, no. 6, pp. 591-596, 2003.

[133] I. A. Clark, "The advent of the cytokine storm," Immunology and Cell Biology, vol. 85, no. 4, pp. 271-273, 2007.

[134] L. Qin, X. Wu, M. L. Block et al., "Systemic LPS causes chronic neuroinflammation and progressive neurodegeneration," Glia, vol. 55, no. 5, pp. 453-462, 2007.

[135] Z. D. Ling, Q. Chang, J. W. Lipton, C. W. Tong, T. M. Landers, and P. M. Carvey, "Combined toxicity of prenatal bacterial endotoxin exposure and postnatal 6-hydroxydopamine in the adult rat midbrain," Neuroscience, vol. 124, no. 3, pp. 619-628, 2004. 


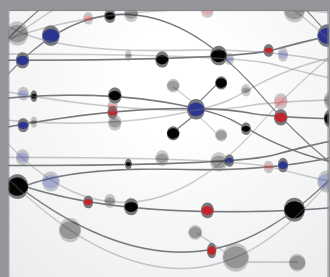

The Scientific World Journal
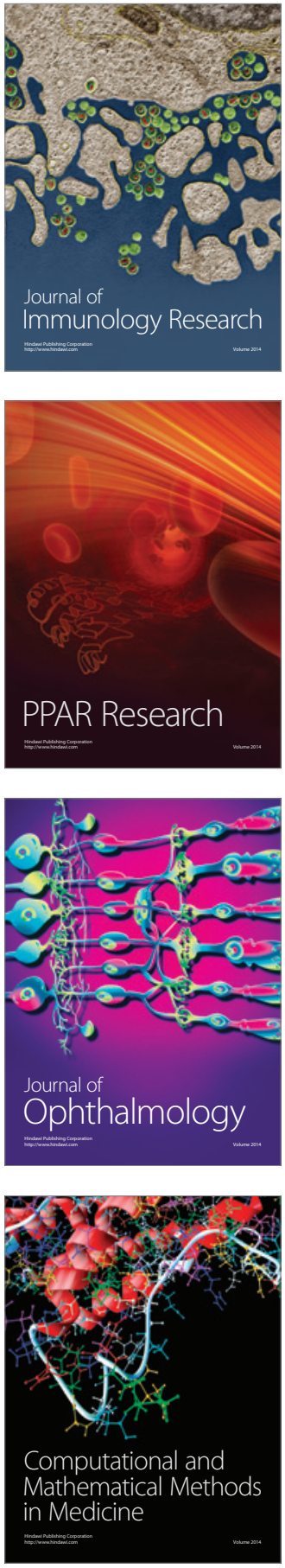

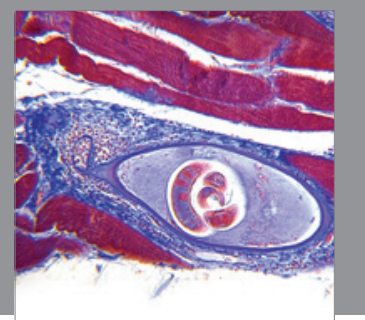

Gastroenterology

Research and Practice
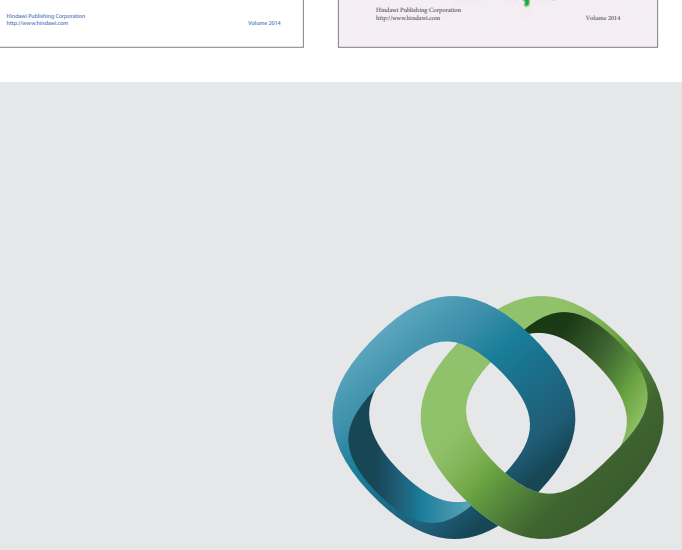

\section{Hindawi}

Submit your manuscripts at

http://www.hindawi.com
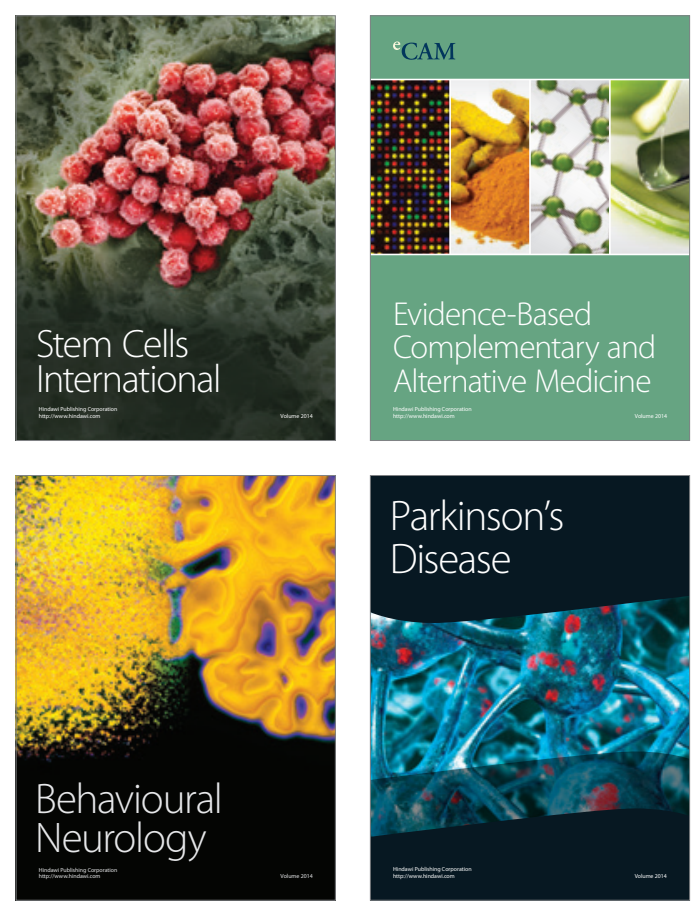

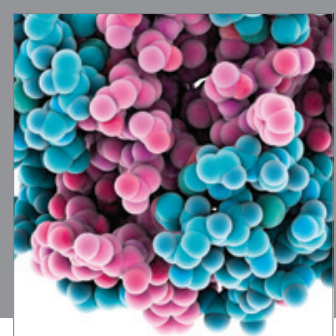

Journal of
Diabetes Research

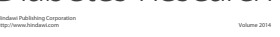

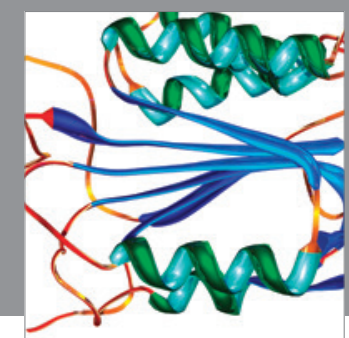

Disease Markers
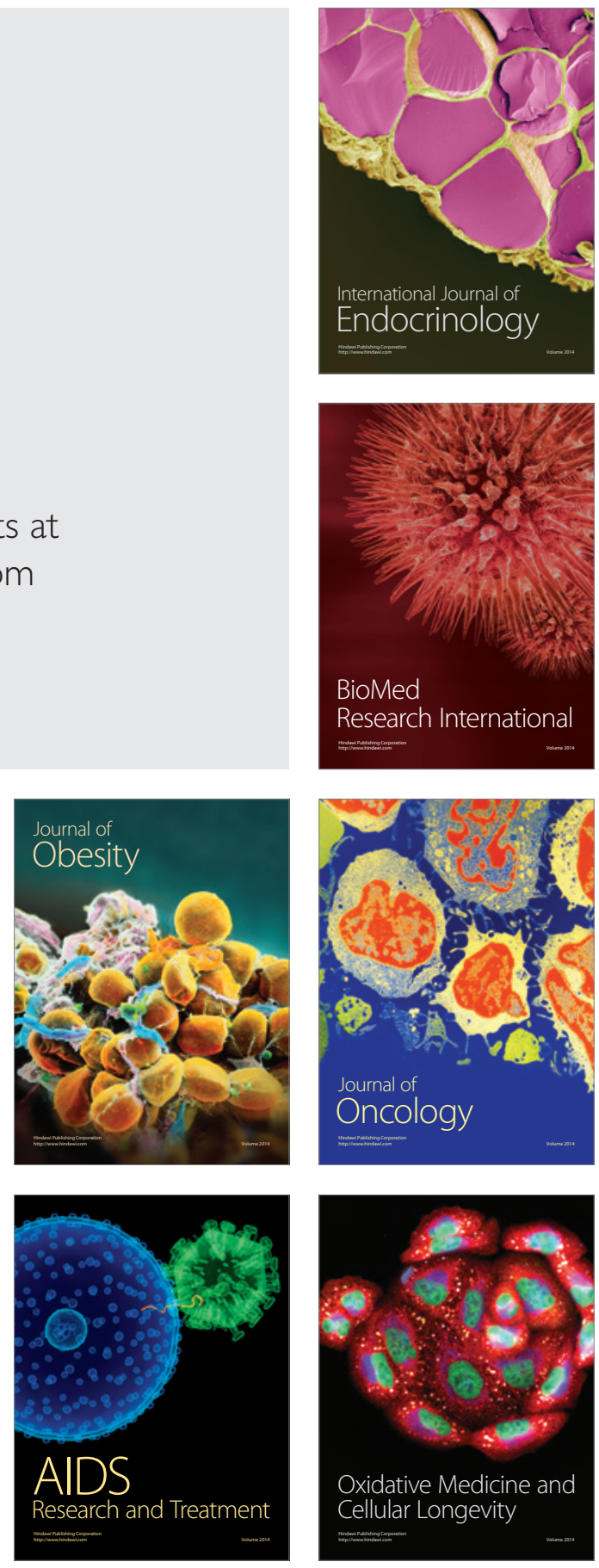